\title{
AN OPTIMIZATION MODEL FOR LINEAR PROJECT SCHEDULING CONSIDERING MULTI-SKILLED CREWS
}

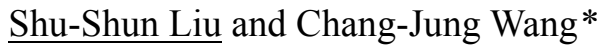 \\ Construction Engineering, National Yunlin University of Science \& Technology, Yunlin, Taiwan \\ * Corresponding author (g9210817@yuntech.edu.tw)
}

\begin{abstract}
Owing to the characteristics of construction linear projects, work efficiency is always addressed by prior researchers. However, most studies only consider single-skilled crews for linear projects, and the flexibility of multiskilling in construction is neglected. Recently, researchers have reveals that multiskilling in construction can increase the productivity, flexibility, and continuity of work, this study therefore proposed a duration optimization mode while introducing the concept of multiskilling to integrate single/multiple skilled crews to improve work performance. Moreover, to enhance the efficiency of problem solving, constraint programming (CP) is employed to handling the complicated combinatorial scheduling problem, and several heuristic rules are engaged. The CP-based optimization is proceeded to minimize project duration, while two crew types, including single-skilled and multi-skilled crews, are integrated in the proposed model. Furthermore, a bridge example is used, and a scenario is conducted and analyzed to illustrate the proposed model for handling linear scheduling problems. Consequently, multiskilling in construction for optimizing project duration and improving work efficiency is demonstrated.
\end{abstract}

Keywords: Linear Project Scheduling, Multi-skilled, Constraint Programming

\section{EXTENDED ABSTRACT}

Due to the characteristics of linear projects, numerous specified scheduling techniques are developed for the linear scheduling projects, including LOB, VPM, LSM, and RSM. However, most research assumes activities are performed using single-skilled crews, and the flexibility of multi-skilling in construction in assigning tasks 1] is neglected. Therefore, this study introduces the concept of multiskilling to integrate single/multiple skilled crews for linear scheduling problems, and provide possible improvement for work performance. Each activity should be executed by one single-skilled crew at least, and one multi-skilled crew may be engaged to shorten work time for the activity if necessary. Based on prior research, the one single-skilled crew is selected and used to complete repetitive activities with the same type. This study complies with the ideal, and moreover introduces the concept of temporary hiring of multi-skilled crews to facilitate work efficiency. That is, one single-skilled crew is employed for one specified activity type, and multi- skilled crews are hired temporarily to improve productivity for activities which are executed by the single-skilled crew already. Moreover, the multi-skilled crew is released while completing the activity, and can be used by other following activities.

In order to enhance the efficiency of problem solving, constraint programming (CP), a computer implementation designed for solving constraint satisfaction problems (CSPs), is employed to handling the complicated combinatorial scheduling problem. Figure 1 illustrates the framework of this study for dealing with construction linear scheduling problems. Two crew types are integrated in the proposed model, including single-skilled and multiskilled crews and the CP-based optimization is proceeded for minimization of project duration. 


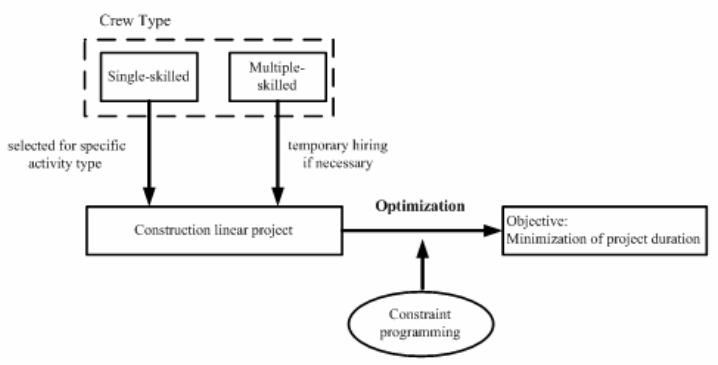

Fig. 1. Optimization framework for linear scheduling Owing to the highly complicated combinatorial problems associated with crew selection and scheduling, this research employs constraint programming (CP) to construct the proposed optimization model. This study adopts CP for model formulation which offers three advantages for the problem domain: (1) The setting of binary variables is clear because of the logical and sequential constraints provided by $\mathrm{CP}$; (2) CP is a naturally appropriate tool for solving combinatorial scheduling problems; and (3) CP-based models are flexible, and various heuristic rules can be easily modified to meet user requirements without rebuilding the model. Based on the above, the proposed $\mathrm{CP}$-formulated optimization model can handle the linear scheduling problems, and identify the optimal solution with various multi-skilled crew selections. Model formulation of this study considers activity relationships, linear scheduling logic, cost calculation, single-skilled crew selection, and heuristic rule for multiskilled crew selection with binary variables. Traditionally, four typical activity relationships are used in construction projects, including finish-start (FS), start-start (SS), finishfinish (FF), and start-finish (SF).Primarily, activity duration depends on by work quantities and productivity of chosen crew for executing. For each activity, at least one main single-skilled crew has to be determined, and one multi-skilled crew may be chosen for improving work if necessary. Hence, binary variables are used to specify the existence of multi-skilled crew. Owing to flexibilities of CP, several heuristic rules can be integrated into the model formulation to enhance the problem solving for multiskilled crew selection. If a multi-skilled crew is used for a specified activity, its corresponding binary variable is one. Furthermore, if its execution time of the activity overlaps the successor, their multi-skilled crews have to be different to each other. Otherwise, if multi-skilled crew is not employed for an activity, the binary variable equals zero. To assist contractors in handling linear scheduling problems and improving work efficiency, this study introduce the concept of multiskilling to meet practical needs, such as duration compression. The objective is therefore shown as the following:

\section{Minimize $T$}

For illustrating the proposed optimization model, a bridge project example employed by prior research [2] is used in this study for scenario analysis. The project consists of five types of repetitive activity, including excavation, foundation, column, beam and slab. Work quantity of each activity, crew's output, and the possible duration/cost for each individual activity with various single-skilled crew selections are complying with the research. Moreover, the information of five multi-skilled crews is assigned, including work type, output and, daily cost.

The analysis result demonstrates the model ability for enhancing efficiency and shortening duration with various multi-skilled crew selections. The optimization duration is shortened from 106.8 to 91.1 days, about 15.7 days. In addition, selection of single/multiple skilled crews is presented. The critical work efficiency issue for linear scheduling problems is therefore recognized for contractors, and the proposed model with the usage of multi-skilled crews is illustrated clearly in the scenario. Consequently, an appropriate project schedule with crew hiring can be identified using the proposed model for fulfilling contractors' needs on linear scheduling problems.

\section{REFERENCES}

[1] Burleson, R.C., Haas, C.T., Tucker, R.L., and Stanley, A., "Multiskilled Labor Utilization Strategies In Construction", Journal of Construction Engineering and Management, Vol. 124 (6), pp. 480-489, 1998.

[2] El-Rayes, K. and Moselhi, O. "Optimizing Resource Utilization For Repetitive Construction Projects", Journal of Construction Engineering and Management, Vol. 127 (1), pp. 18-27, 2001. 